Original Research

\title{
A bone replacement-type calcium phosphate cement that becomes more porous in vivo by incorporating a degradable polymer
}

\author{
Akiyoshi Shimatani ${ }^{1} \cdot$ Hiromitsu Toyoda $\mathbb{D}^{1} \cdot$ Kumi Orita $^{1} \cdot$ Yuta $_{\text {Ibara }}{ }^{2} \cdot$ Yoshiyuki Yokogawa $^{2} \cdot$ Hiroaki Nakamura $^{1}$
}

Received: 30 January 2021 / Accepted: 7 June 2021 / Published online: 22 June 2021

(C) The Author(s) 2021

\begin{abstract}
This study investigated whether mixing low viscosity alginic acid with calcium phosphate cement (CPC) causes interconnected porosity in the CPC and enhances bone replacement by improving the biological interactions. Furthermore, we hypothesized that low viscosity alginic acid would shorten the setting time of CPC and improve its strength. CPC samples were prepared with $0,5,10$, and $20 \%$ low viscosity alginic acid. After immersion in acetate buffer, possible porosification in CPC was monitored in vitro using scanning electron microscopy (SEM), and the setting times and compressive strengths were measured. In vivo study was conducted by placing CPC in a hole created on the femur of New Zealand white rabbit. Microcomputed tomography and histological examination were performed 6 weeks after implantation. SEM images confirmed that alginic acid enhanced the porosity of CPC compared to the control, and the setting time and compressive strength also improved. When incorporating a maximum amount of alginic acid, the new bone mass was significantly higher than the control group $(P=0.0153)$. These biological responses are promising for the translation of these biomaterials and their commercialization for clinic applications.
\end{abstract}

\section{Graphical Abstract}

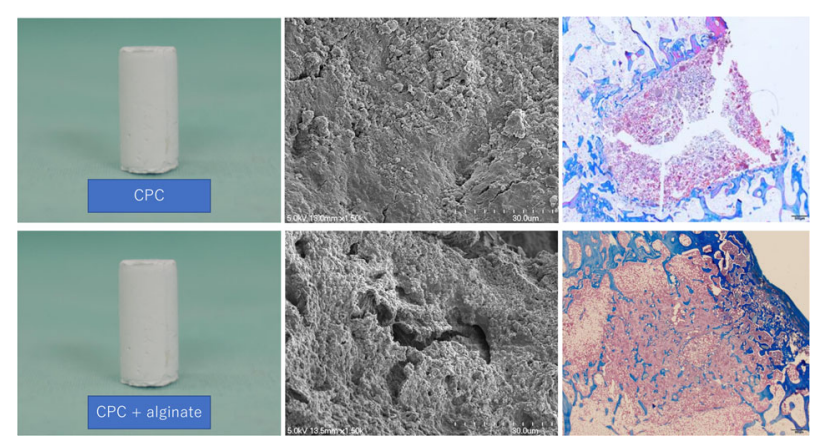

Akiyoshi Shimatani

bpcip920@tcct.zaq.ne.jp

1 Department of Orthopedic Surgery, Graduate School of Medicine, Osaka City University, 1-4-3 Asahi-Machi, Abeno-ku, Osaka 5458585, Japan

2 Department of Mechanical \& Physical Engineering, Graduate School of Engineering, Osaka City University, 3-3-138 Sumiyoshi-ku, Osaka 558-8585, Japan

\section{Introduction}

The use of calcium phosphate cements (CPCs) has increased in recent years, because they are useful in the direct filling of bone defects and as cell matrices in tissue engineering [1, 2]. Compared to other candidate bone replacement materials, the main merit of CPCs is their selfsetting nature, which is particularly helpful when employed in the injectable form [3]. Nevertheless, CPCs suffer from several critical issues [4]. Self-setting CPCs generally have a dense microstructure. The lack of pores slows down the degradation by osteoclast-dependent resorption, which limits the potential of tissue ingrowth [5, 6]. Another main challenge is their generally poor mechanical properties [4]. 
Table 1 Composition of CPC

\begin{tabular}{lllll}
\hline & Liquid 0 (CPC0) & Liquid 1 (CPC1) & Liquid 2 (CPC2) & Liquid 3 (CPC3) \\
\hline Milli-Q water $(\mathrm{g})$ & 10 & 10 & 10 & 10 \\
Alginic acid $(\mathrm{g})$ & 0 & 0.5 & 1 & 2 \\
K2HPO4 (g) & 0.2 & 0.2 & 0.2 & 0.2 \\
KH2PO4 (g) & 0.1 & 0.1 & 0.1 & 0.1 \\
\hline
\end{tabular}

To attain better mechanical and biological properties, many researchers have combined CPC with degradable polymers. Widely studied biopolymers include gelatin, collagen, chitosan, and alginate [7-11]. These biopolymers could accelerate the setting reaction while enhancing the strength of the CPC network. The setting time is especially important in clinical use [12]. The cure time of CPC is also $\mathrm{pH}$ dependent, and premature solidification has been reported under acidic conditions [13].

This study evaluated a series of CPC-alginate compounds. Alginate is a naturally derived polysaccharide that has been used in many biomedical applications, including cell encapsulation, drug delivery, and wound dressing because of its nontoxicity, degradability, and cross-linking ability [11, 14]. However, compared to other biopolymers, there have been only limited studies of alginate in conjunction with CPCs [10, 15], particularly for systematic investigation of the mechanical properties and in vivo performance. Furthermore, we are not aware of any previous experiments that considered enhancing CPC with low viscosity alginic acid, which has a much higher solubility than the medium viscosity one.

The present study aims to incorporate low viscosity alginic acid into CPC as a self-setting bioactive cement. The composition, morphology/microstructure, setting time, compressive strength, surface reaction, and degradation of CPC-alginate composites and the associated bone healing were investigated in vitro and in vivo. Our main hypothesis is that the introduction of low viscosity alginic acid causes the formation of a series of interconnected macropores that facilitate the entry of bone marrow cells and new bone formation.

\section{Materials and methods}

\subsection{In vitro study and characterization}

\subsubsection{Preparation of CPC-containing polymer complex}

Tetracalcium phosphate (TTCP) powder was synthesized as follows. A calcium hydroxide solution (Nacalai Tesque Inc., Japan) was added dropwise into a phosphoric acid solution (Wako Pure Chemical Industries, Ltd., Japan) that was kept below $10^{\circ} \mathrm{C}$, and the solution was stirred at room temperature for $24 \mathrm{~h}$. The precipitate was filtered, dried, and heated at $1500{ }^{\circ} \mathrm{C}$ for $5 \mathrm{~h}$, ground in an agate mortar, and then sieved under $75 \mu \mathrm{m}$ to obtain the TTCP powder (average particle size: $21.5 \mu \mathrm{m}$ ). Commercial dicalcium phosphate anhydrous (DCPA) powder (Taihei Chemical Industrial Co., Ltd., Japan) was ground in a ball mill with pure water and zirconia balls for $48 \mathrm{~h}$ to obtain the DCPA powder (average particle size: $0.52 \mu \mathrm{m}$ ). Equal molars of TTCP powder and DCPA powder were mixed in a blender and with an agate mortar to form the CPC powder.

A mixed solution of dipotassium hydrogen phosphate and potassium dihydrogen phosphate $(0.2$ and $0.1 \mathrm{~g}$, respectively, both from Kishida Chemical Co., Ltd., Japan) was prepared with $10 \mathrm{~mL}$ of Milli-Q water. Low viscosity sodium alginate (UIV-L3, viscosity $20-50 \mathrm{mPa} \cdot \mathrm{s}$, KIMICA Co., Japan) was used as a biocompatible polysaccharide and gelation agent to develop the nonrigid CPC. Four liquids (Liquid 0 to Liquid 3) were prepared with 0, 5, 10 , and $20 \mathrm{wt} \%$ low viscosity sodium alginate, respectively. The CPC powder was mixed with each curing liquid at a powder/liquid ratio of 3 , and the mixture was placed in a cylinder (Table 1). The corresponding hardened specimens were denoted CPCO-CPC3.

\subsubsection{Characterization}

The $\mathrm{pH}$ of the prepared curing liquid was measured using a pH meter (D-51, HORIBA, Ltd., Japan). The pH meter was first calibrated using three points measured in phosphate $\mathrm{pH}$ standard solution ( $\mathrm{pH} 9.18$ ), neutral phosphate $\mathrm{pH}$ standard solution ( $\mathrm{pH}$ 6.86), and phthalate $\mathrm{pH}$ standard solution $(\mathrm{pH}$ 4.01), all from Wako Pure Chemical Industries, Ltd., Japan. Five replicates were measured for each liquid, and the average value is reported.

The setting time was measured according to JIS (Japanese Industrial Standards) T0330-4. Kneaded CPC paste was placed in a cylindrical Teflon mold (inner diameter: $6 \mathrm{~mm}$, height: $12 \mathrm{~mm}$ ) within $90 \mathrm{~s}$ after the start of kneading. The mold was placed in a container that also held a sponge soaked in Milli-Q water in an incubator held at $37{ }^{\circ} \mathrm{C}$. Setting of the material was monitored using a Vicat needle (A-004, MECC Co., Ltd, Japan) with a $1 \mathrm{~mm}^{2}$ tip at $300 \mathrm{~g}$ load. Every $30 \mathrm{~s}$, the Vicat needle was placed on the 


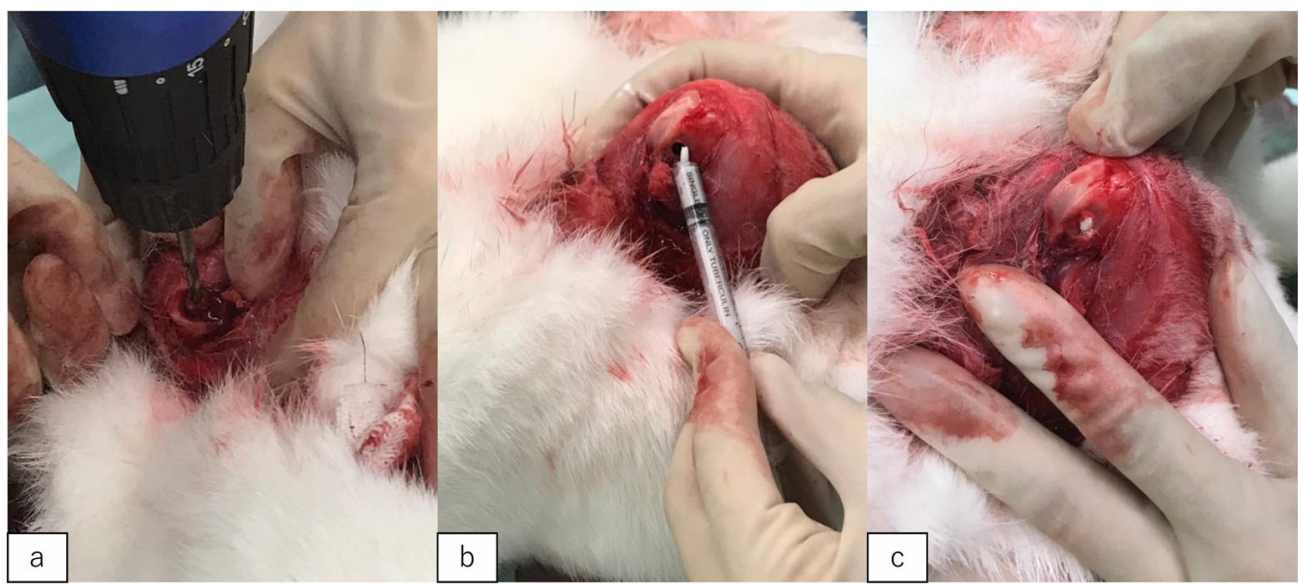

Fig. 1 Photographs of the operation. a Preparation of a bone hole (diameter: $4 \mathrm{~mm}$, depth: $10 \mathrm{~mm}$ ) outside the condyle of the rabbit femur. b, c Material was injected into the bone hole using a syringe

surface of the specimen, and the specimen was considered set when the needle failed to make a perceptible circle on its surface. The test was performed in triplicates for each condition, and the average value was reported as the setting time.

The compressive strength was determined in accordance with JIS T0330-4. The mixture of CPC powder and liquid was placed in the hole of a Teflon mold (diameter: $6 \mathrm{~mm}$, height: $12 \mathrm{~mm}$ ). The mold was stored in an incubator at $37^{\circ} \mathrm{C}$ and $100 \%$ humidity for $1 \mathrm{~h}$, and then immersed in Milli-Q water for 1 day. The diameter of each specimen was measured using a micrometer (M300, Mitsutoyo, Japan). Then, the specimen was individually placed on the stage of a universal tester (Autograph AGI-20kN, Shimadzu Co., Japan). Measurement was carried out at a head speed of $0.500 \mathrm{~mm} \mathrm{~s}^{-1}$ and a sampling interval of $100 \mathrm{~ms}$. Five specimens from each type of CPC were used, and each specimen was measured ten times. Data analysis was performed using control/analysis software (Trapezium, Shimadzu Co., Japan), and the average value was used as the compressive strength.

Scanning electron microscopy (SEM) analysis employed an S-4700 (Hitachi Ltd., Japan) instrument at an accelerating voltage of $5.0 \mathrm{kV}$ under a pressure of $1.00 \times$ $10^{-3} \mathrm{~Pa}$ or less, on samples coated with $12 \mathrm{~nm}$ of Pt-Pd using an ion sputter (E-1030, Hitachi Ltd., Japan). The mixture of CPC powder and liquid was placed in a cylindrical Teflon mold (diameter: $6 \mathrm{~mm}$, height: $12 \mathrm{~mm}$ ). Each mold was stored in an incubator at $37^{\circ} \mathrm{C}$ and $100 \%$ humidity for $1 \mathrm{~h}$, and then immersed in acetic acid-sodium acetate buffer (see details below) for 7 days. The diameter of each specimen was measured using a micrometer (M300, Mitsutoyo, Japan). For the buffer, first two solutions were prepared using Milli-Q water: one containing
$0.08 \mathrm{~mol} \mathrm{~L}^{-1}$ acetic acid (Kishida Chemical Co., Ltd. Japan) and the other containing $0.08 \mathrm{~mol} \mathrm{~L}^{-1}$ sodium acetate (Kishida Chemical Co., Ltd., Japan). Then, the two solutions were mixed at a ratio of 1:7 to prepare the buffer, which had a pH of $5.50 \pm 0.02$.

\subsection{In vivo studies}

\subsubsection{Surgery}

The animal protocol was approved by the Animal Ethics Committee of Osaka City University. Ten retired female New Zealand white rabbits weighting 4-4.5 kg were used for implantation of $\mathrm{CPC} 0$ (control), CPC1, CPC2, and $\mathrm{CPC} 3$. Five femurs were used in each group. The rabbits were anesthetized with ketamine hydrochloride, and a cylindrical defect (diameter: $4 \mathrm{~mm}$, depth: $10 \mathrm{~mm}$ ) was drilled on both femur condyles using a trephine drill. A CPC sample was injected into the defect site, which was then sutured (Fig. 1). After 6 weeks, the rabbits were sacrificed, and the femur condyles were obtained for analysis. The surgery was performed by two authors (AS and $\mathrm{KO})$.

\subsubsection{X-ray and micro-CT analysis}

$\mathrm{X}$-ray images were taken for each femur condyle immediately after surgery as well as 3 and 6 weeks later. The extracted rabbit femur condyles were fixed in $10 \%$ neutral buffered formation solution at room temperature. Micro-CT was done using inspeXio SMX-90CT Plus (Shimadzu Corporation, Japan). The scan data were reconstructed using 3D image processing software (ExFact VR, Nihon Visual Science, Inc., Japan). 


\subsubsection{Histological analysis}

After the micro-CT analysis, each sample was decalcified with Morse solution (Wako Pure Chemical Industries, Ltd., Japan) and dehydrated using an alcohol series. Residual alcohol was removed by immersion in xylene. After embedding the tissue sample in paraffin block, 4- $\mu$ m-thick slices were cut using a microtome and treated with hematoxylin and eosin (H\&E) and Masson's trichrome stains. The sections were observed using a model BX53F microscope (Olympus, Japan) and photographed with an Olympus DP74 camera. The images were analyzed using Cellsens software (Olympus, Japan). The area of new bone mass was measured using ImageJ based on Masson's trichrome staining. The total area of new bone was measured in a 100-fold visual field at three locations: on the cortical bone side, the central side, and the cancellous bone side of the bone defects. Image analysis and measurement of the total area of new bone were conducted by two authors (HT and $\mathrm{HN}$ ) in a blinded situation.

\subsection{Statistical analysis}

Statistical analysis was performed using the Excel Statistics software for Windows (version 2019; SSRI Co. Ltd., Tokyo, Japan). Data were expressed as mean \pm standard deviation. The data analysis used one-way analysis of variance followed by a multiple comparison using the Tukey test. $P$ values $<0.05$ were considered statistically significant.

\section{Results}

\subsection{In vitro properties}

Table 2 shows the $\mathrm{pH}$, setting time, and compressive strength for different curing liquids or CPCs. The $\mathrm{pH}$ of the curing liquids was significantly reduced after adding sodium alginate. Liquid 3 with $20 \mathrm{wt} \%$ alginic acid showed the lowest $\mathrm{pH}(7.00 \pm 0.05)$, which is statistically significantly lower than that of Liquid $0(7.21 \pm 0.05, P<$ 0.001). At the same time, the setting time of CPC was shortened with increasing content of alginic acid, from $56 \pm$ $4.6 \mathrm{~min}$ for $\mathrm{CPC} 0$ to $11.5 \pm 0.5 \mathrm{~min}$ for $\mathrm{CPC} 3$. Therefore, the curing liquid with the lowest $\mathrm{pH}$ (Liquid 3) resulted in the shortest setting time, with statistically significant difference compared to the control $(P<0.001)$. The compressive strength also significantly increased after adding sodium alginate compared to the control. CPC 3 showed the highest compressive strength of $46.7 \pm 8.6 \mathrm{MPa}$, whereas CPC0 showed the lowest $(7.3 \pm 2.1 \mathrm{MPa})$. Statistically, CPC3 was significantly stronger than CPC0 $(P<0.001)$.

Figure $2 \mathrm{a}$ is a representative low-magnification SEM image of $\mathrm{CPC} 0$, and Fig. 2a1 is the high-magnification counterpart. The corresponding images for $\mathrm{CPC} 3$ are displayed in Fig. 2b and Fig. 2b1, respectively. In the lowmagnification image, the alginic acid-containing CPC3 shows more pores and a lower density than the control (CPC0). The high-magnification images further indicate that the $\mathrm{CPC} 3$ polymer matrix has more large pores than $\mathrm{CPC}$.

\subsection{In vivo results}

Representative X-ray images are presented in Fig. 3. Defect bridging was monitored by serial radiography at 0,3 , and 6 weeks after surgery. At 3 weeks, we observed more elaborate structures and extensive absorption at the defect site treated with CPC3 compared with the other groups. At 6 weeks, bone regeneration of marrow space was visible in the group treated with CPC3.

Six weeks after the operation, the rabbits were sacrificed, and all treated femurs were imaged by micro-CT. Similar to the X-ray images, no CPC degradation was observed in the CPC0 group, whereas the CPC 3 group showed degradation and bone formation at the defect periphery. This result suggests that early bone replacement occurred, and there may be bone ingrowth into the CPC 3 implant at 6 weeks (Fig. 4).

Figures 5 and 6 display representative images of sections stained with H\&E and Masson's trichrome stain, respectively. After 6 weeks, the accumulation of nucleated cells around the $\mathrm{CPC} 0$ implant was poor. In contrast, the implants containing alginate (CPC1-CPC3) showed accumulation of nucleated cells and new bone formation around them. Most of the gaps in CPC0 were covered with fibrous tissue, however in CPC1-CPC3 there was much collagen tissue (Fig. 5). Complete cortical bridging was observed in $\mathrm{CPC} 1-\mathrm{CPC} 3$, while no apparent cortical bridging was
Table $2 \mathrm{pH}$, setting time and compressive strength

\begin{tabular}{lcccc}
\hline & Liquid 0 (CPC0) & Liquid 1 (CPC1) & Liquid 2 (CPC2) & Liquid 3 (CPC3) \\
\hline $\mathrm{pH}$ & $7.21 \pm 0.05$ & $7.05 \pm 0.04^{*}$ & $7.11 \pm 0.03^{*}$ & $7.00 \pm 0.05^{*}$ \\
Setting time (min) & $56 \pm 4.6$ & $20 \pm 1.7^{*}$ & $17 \pm 2.6^{*}$ & $11.5 \pm 0.5^{*}$ \\
Compressive strength $(\mathrm{MPa})$ & $7.3 \pm 2.1$ & $16.3 \pm 3.7$ & $34.9 \pm 4.1^{*}$ & $46 . .7 \pm 8.6^{*}$ \\
\hline
\end{tabular}

$* P<0.05$, each liquid/CPC group versus Liquid 0/CPC0 group 
Fig. 2 SEM micrographs. Crosssectional microstructures in the set CPC samples a without and b with alginate $(20 \mathrm{wt} \%)$. Little porosity was detected in CPC sample without alginate (a1). Adding alginate resulted in the formation of macropores within the bulk materials (b1)

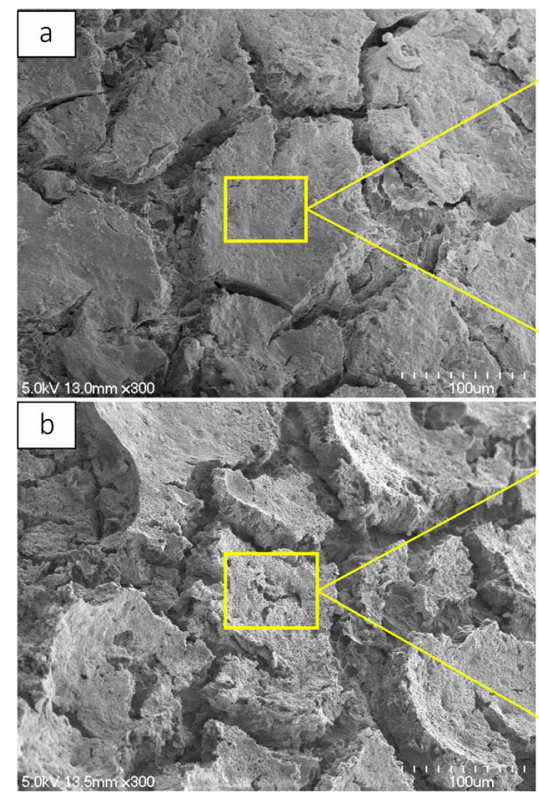

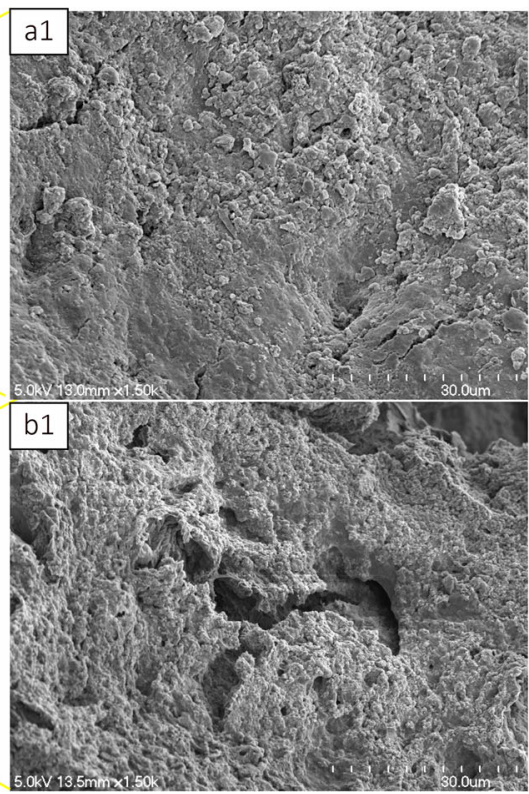

(a) $\mathrm{CPCO}$
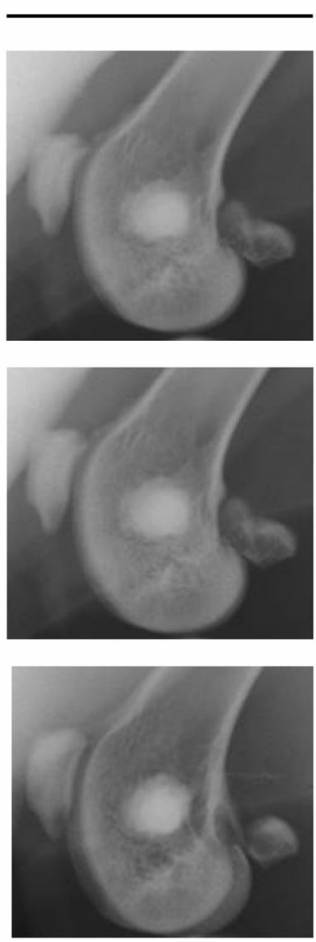

(b) $\mathrm{CPC1}$
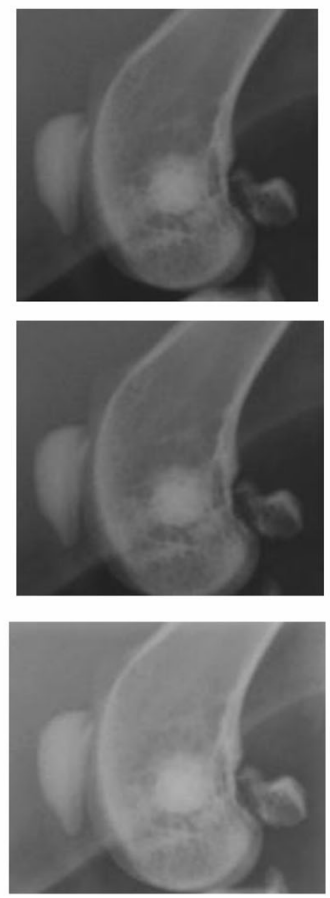

(c) $\mathrm{CPC} 2$
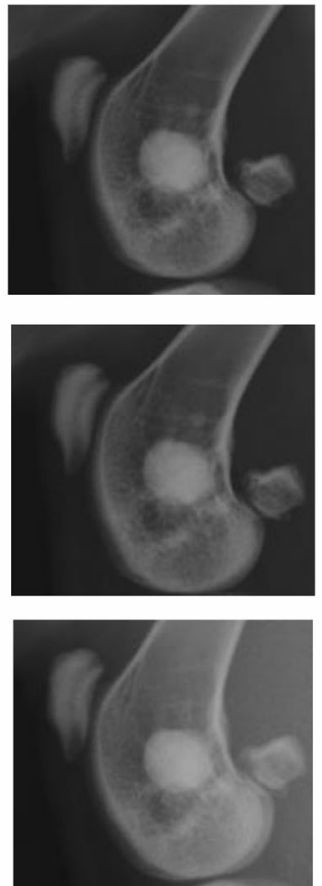

(d) $\mathrm{CPC3}$
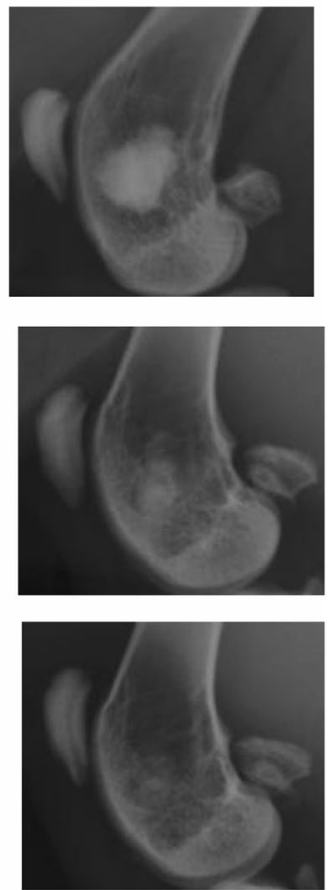

Fig. 3 X-ray images of CPCs at 0 day, 3 weeks, and 6 weeks after surgery. Impaired portion of femoral condyle indicates the CPCs. Only CPC3 displays a time-dependent increase in permeability at the site. This is especially noticeable in the image after 6 weeks

observed in CPC0. CPC3 further showed apparent new bone formation within the implant (Fig. 6).

According to the area of new bone in the bone hole measured after Masson's trichrome staining, CPC3 resulted in a larger amount of new bone than the CPC0 group with statistical significance $(P=0.0153$, Fig. 7).

\section{Discussion}

This study demonstrated that the incorporation of low viscosity alginic acid caused a porous CPC (Fig. 2). Moreover, the setting time was reduced and the compressive strength increased (Table 2). When this composite was implanted in an 
animal model, there was extensive new bone formation with gradual degradation of CPC (Figs. 4-7). This is the first study to show that low viscosity alginic acid enhances the biological performance of CPCs.

(a) $\mathrm{CPCO}$
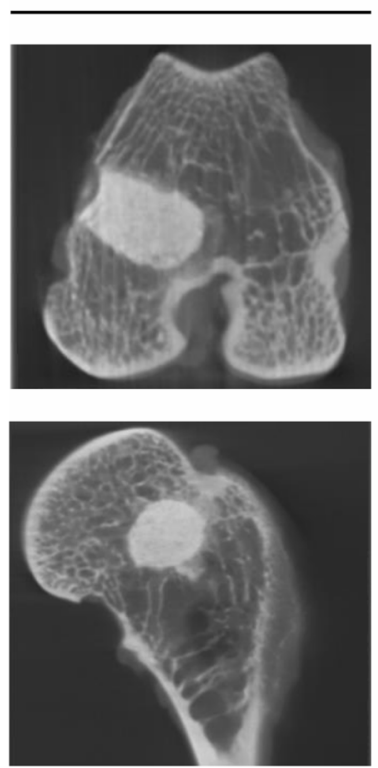

(b) CPC3
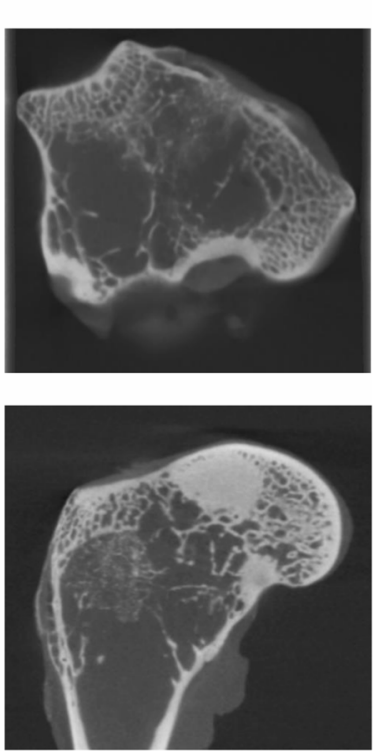

Fig. 4 Micro-CT images of CPC0 and CPC3. Upper part: axial image, lower part: sagittal image. CPC3 shows lower brightness in the bone tunnel than $\mathrm{CPCO}$ (b) $\mathrm{CPC1}$

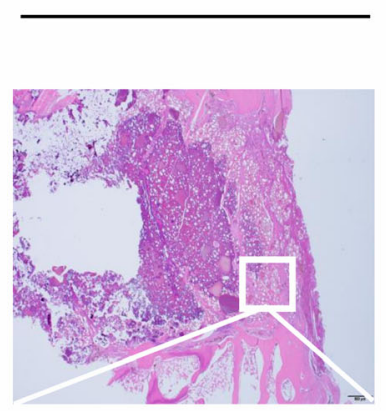

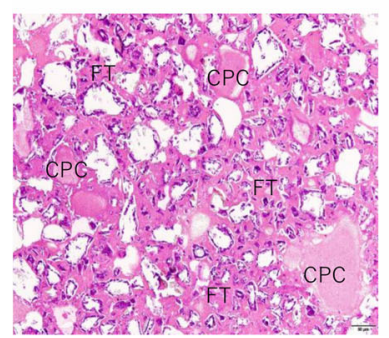

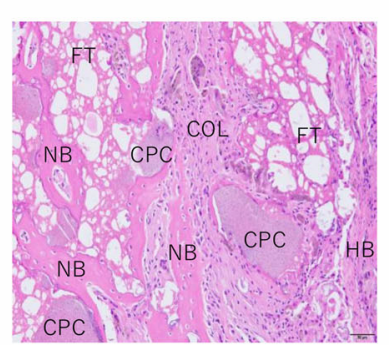

Fig. 5 Histological micrographs of tissue sections stained with hematoxylin and eosin. Implant interface of $\mathrm{CPC} 0-\mathrm{CPC} 3$ after 6 weeks of implantation in rabbit femoral condyle. The images show
Injectable CPC is widely used for repairing bone defect and bone augmentation due to the minimal invasion required [16, 17]. A number of polymers (chitosan, gelatin, hyaluronic acid, methylcellulose, and others) have been added as viscous binders to improve the biological performance of CPC materials and their injectability [18]. In this study, we investigated the effect of adding different amounts of low viscosity sodium alginate on the CPC's setting time, mechanical properties in vitro, and the biocompatibility and bone replacement rate in vivo (rabbit femur). From the results, the injectable CPC 3 composite containing $20 \mathrm{wt} \%$ alginate showed degradation and subsequent bone replacement, with significant advantages over pure CPC and other composites with less alginate.

Specifically, we used a low viscosity sodium alginate (viscosity $20-50 \mathrm{mPa} \cdot \mathrm{s}$, molecular weight about 40,000-50,000). As far as we know, low viscosity alginic acid has not been applied in the curing liquid for CPC before. When its amount exceeds $20 \mathrm{wt} \%$, the liquid will gel strongly, and kneading it with CPC would be difficult. Thus, the limit was set at $20 \mathrm{wt} \%$. For the more commonly used medium viscosity alginic acid (sodium alginate $80-120$, viscosity $80-120 \mathrm{mPa} \cdot \mathrm{s}$, molecular weight about 64,000, Wako Pure Chemical Industries, Ltd.), the maximum dissolution amount is only $2 \mathrm{wt} \%$ (data not shown). Therefore, a much larger amount of polymer (6.4-7.8 times by weight) could be dissolved when using the low viscosity sodium alginate. (c) $\mathrm{CPC2}$
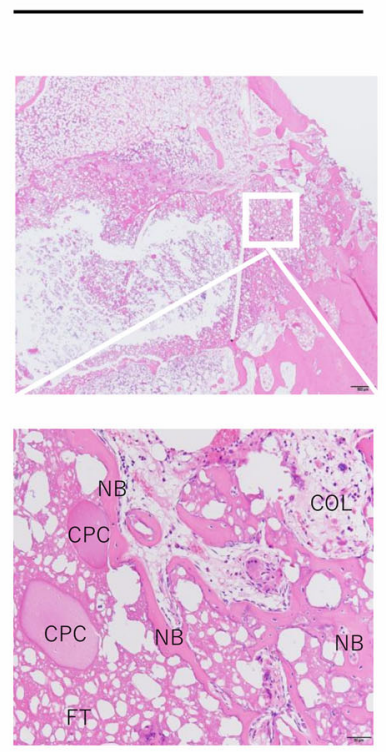

(d) $\mathrm{CPC3}$
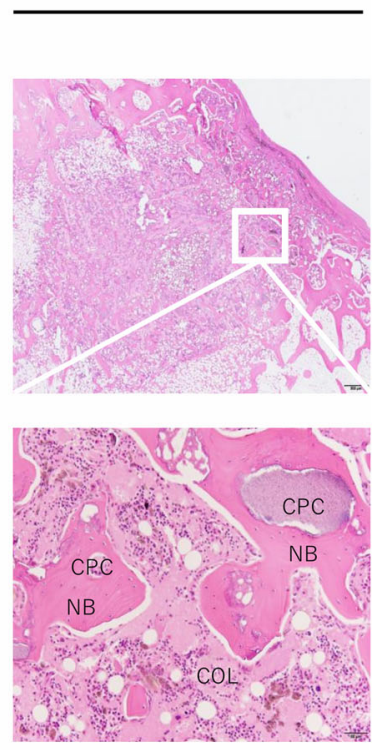

axial cross section through the center of the bone hole. NB newly formed bone, HB host bone, COL collagen, FT fibrous tissue 
(a) $\mathrm{CPCO}$
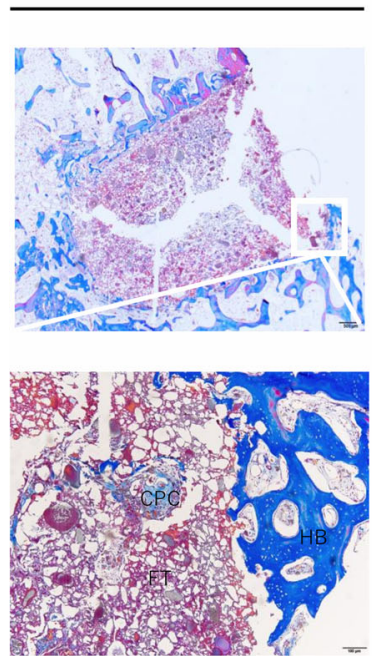

(b) $\mathrm{CPC} 1$
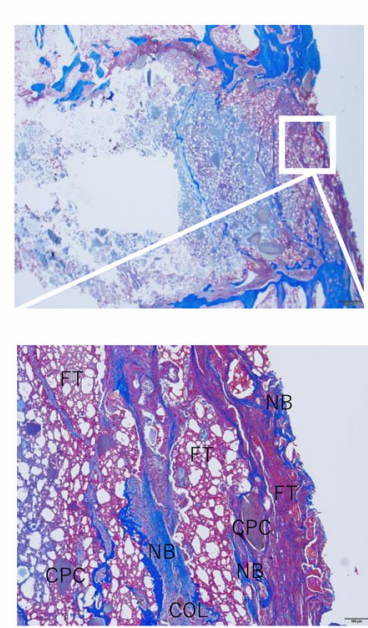

Fig. 6 Histological micrographs of tissue sections stained with Masson's trichrome. Masson's trichrome-stained sections of CPC0-CPC3 after 6 weeks of implantation in rabbit femoral condyle. Images show

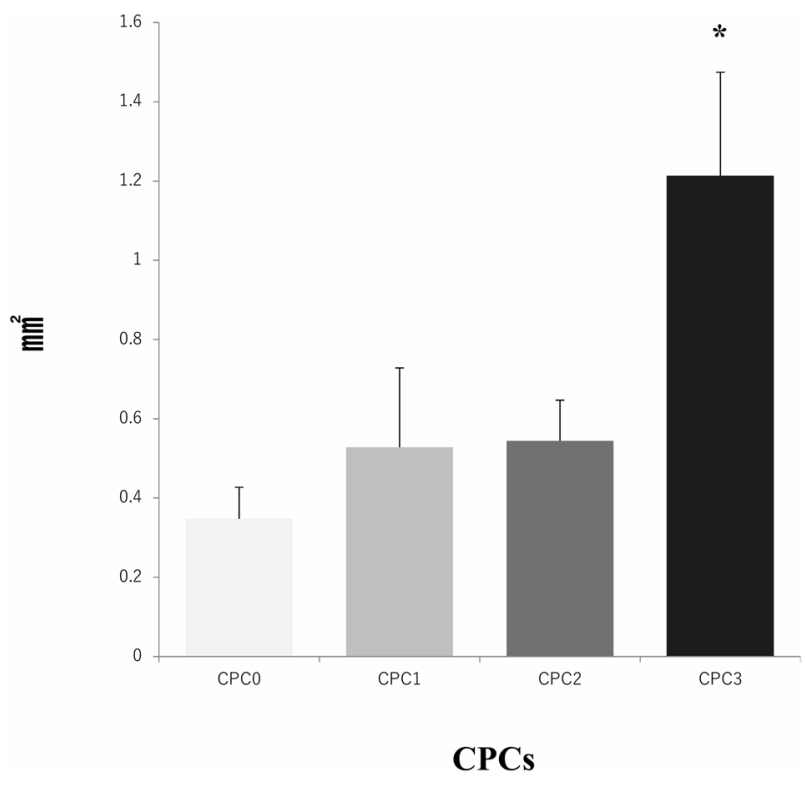

Fig. 7 Area of new bone in the bone tunnel. The area was measured using ImageJ and based on the tissue after Masson's trichrome staining. A higher alginic acid content in the CPC resulted in a larger new bone area. ${ }^{*} \mathrm{CPC} 3$ had a statistically significant increase in compression strength compared to $\mathrm{CPC} 0(P=0.0153)$

Adding sodium alginate to CPC produced several benefits. First, it reduced the average setting time from $56 \mathrm{~min}$ in $\mathrm{CPC} 0$ to $11.5 \mathrm{~min}$ in $\mathrm{CPC} 3$, representing a reduction of $80 \%$. In actual surgeries, a shorter curing time is advantageous [19]. It has been reported that under acidic conditions, the solubility of CPC increases and premature solidification of CPC is likely [13]. In this study, we also observed a lower $\mathrm{pH}$ when increasing the content of alginic (c) $\mathrm{CPC} 2$

(d) $\mathrm{CPC3}$
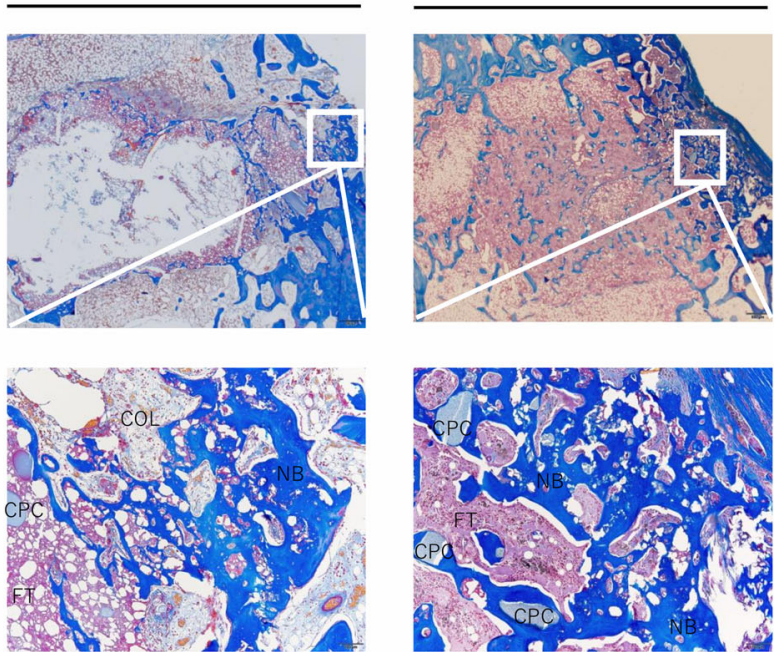

axial cross section through the center of the bone hole. NB newly formed bone, HB host bone, COL collagen, FT fibrous tissue

acid (possibly due to its chelating effect), which is thought to shorten the setting time. The setting of CPC proceeds via dissolution-precipitation reactions, similar to the setting reactions of gypsum [20-22]. In the case of $\alpha$-tricalcium phosphate $\left(\alpha-\mathrm{TCP}, \alpha-\mathrm{Ca}_{3}\left(\mathrm{PO}_{4}\right)_{2}\right)$-based cement, $\alpha$-TCP dissolves to supply $\mathrm{Ca}^{2+}$ and $\mathrm{HPO}_{4}{ }^{2-}$. This liquid phase would be supersaturated with respect to calcium deficient hydroxyapatite (cdHAp; $\mathrm{Ca}_{9}\left(\mathrm{HPO}_{4}\right)\left(\mathrm{PO}_{4}\right)_{5}(\mathrm{OH})$ ), leading to the precipitation of cdHAp. The precipitated cdHAp crystals then interlock with each other to form a set mass. Acceleration of either step mentioned above could theoretically accelerate cdHAp formation, thereby shortening the setting time of CPC [23].

Second, CPC3 with a maximum amount of sodium alginate had a compression strength 6.4 times that of the control (average values: 46.7 versus $7.3 \mathrm{MPa}$ ). It was reported that cdHAp on the surface of $\alpha$-TCP affects not only the setting time but also the mechanical strength of the set CPC. When cdHAp is present on the surface of $\alpha-\mathrm{TCP}$ powder, the $\mathrm{Ca}^{2+}$ and $\mathrm{HPO}_{4}{ }^{2-}$ ions supplied by $\alpha$-TCP would be used for the growth of cdHAp crystals and facilitate their interlocking [23]. The increased strength is attributed to the addition of high molecular weight polymer, as well as ionic cross-linking (a characteristic of alginic acid). This increased strength with added alginic acid is certainly superior to that of cancellous bone (2-12 MPa), but not that of cortical bone (100-230 MPa). Hence, this CPC compound is more suitable for nonloaded surfaces.

Third, 6 weeks after implanting CPC 3 containing $20 \mathrm{wt} \%$ alginic acid, early bone replacement was observed on the $\mathrm{X}$ ray and micro-CT images. The area of new bone was 3.5 
times that of the $\mathrm{CPC} 0$ group. Although $\mathrm{CPC}$ is a convenient injectable bone replacement material, it is difficult to formulate into specific shapes that are useful for tissue engineering [24]. However, Lee et al. reported that a moist environment filled with alginic acid in the CPC space tends to form 3D scaffolds with complex shapes, providing a 3D matrix suitable for tissue cells to adhere and spread [25]. In this study, we also immersed CPC in an acetate-sodium acetate buffer prior to SEM imaging, in order to observe the surface changes in a similar environment. Activated osteoclasts express many proton pumps in the cell membrane on their bone surface side, producing an acidic region with $\mathrm{pH}$ $=$ ca. $4.7-6.8$ on the bone surface [26]. The SEM images confirmed that the alginic acid-containing CPC was more porous than the control. It is thought that the bone replacement action is enhanced when the porosity increases. Also, the porous microstructure allows bone marrow cells to migrate into the CPC composite. Extensive new bone formation with gradual degradation of CPC was observed in this composite. In terms of mechanical strength, we speculate that the slow biodegradation and excellent osteoinductivity are important factors for the synthetic biomaterials to repair bone defects. Such injectable CPC materials containing low viscosity alginic acid may have great potentials in bone regeneration, with significant clinical advantage over the simple CPC. This is an attractive option in the field of bone tissue engineering, especially for the minimally invasive technique. A future technology for mixing any type of alginic acid with the curing solution may allow more precise control over the size of interconnected pores in CPC and its degradation rate. Another interesting possibility is using some kind of degradable polymer loaded with multiple drugs, growth factors, etc. to enhance angiogenesis and osteogenesis in challenging environments such as concomitant infection, poor blood supply, and huge bone defects.

\section{Conclusions}

Low viscosity alginic acid not only possesses good biocompatibility and degradability, but also causes faster and more effective osteogenesis when combined with CPC for bone repair. In this study, combinations of CPC and alginic acid demonstrated a reasonable setting time, suitable mechanical strength, and excellent degradability and bioactivity. Since it is prepared in the paste form, this composite can be easily injected. Such cement could become good artificial bone material for clinical applications.

\section{Data availability}

Data supporting the findings of this study are available within the article.
Acknowledgements The authors thank the staff at the Central Laboratory of Osaka City University Medical School for technical support.

Author contributions Conceptualization: HT and YY; methodology: AS and YI; validation: $\mathrm{KO}$ and $\mathrm{HT}$; formal analysis: AS; investigation: AS and KO; resources: AS; data curation: HT; writing —original draft preparation: AS; writing-review and editing: HT and YY; visualization: $\mathrm{KO}$ and $\mathrm{HT}$; supervision: $\mathrm{HN}$; project administration: $\mathrm{HT}$ and $\mathrm{HN}$; and funding acquisition: HT, YY, and HN. All authors have read and agreed to the published version of the manuscript.

Funding This research was funded by the Japan Society for the Promotion of Science (JSPS) KAKENHI (Grant No. 20K05118). The funders had no role in the design of the study; in the collection, analysis, or interpretation of data; in the writing of the manuscript; or in the decision to publish the results.

\section{Compliance with ethical standards}

Conflict of interest The authors declare no competing interests.

Ethics approval Study approval was obtained from Osaka City University Graduate of Medicine. All investigations were conducted in accordance with the Osaka City University Hospital Ethical Principles of Research.

Publisher's note Springer Nature remains neutral with regard to jurisdictional claims in published maps and institutional affiliations.

Open Access This article is licensed under a Creative Commons Attribution 4.0 International License, which permits use, sharing, adaptation, distribution and reproduction in any medium or format, as long as you give appropriate credit to the original author(s) and the source, provide a link to the Creative Commons license, and indicate if changes were made. The images or other third party material in this article are included in the article's Creative Commons license, unless indicated otherwise in a credit line to the material. If material is not included in the article's Creative Commons license and your intended use is not permitted by statutory regulation or exceeds the permitted use, you will need to obtain permission directly from the copyright holder. To view a copy of this license, visit http://creativecommons. org/licenses/by/4.0/.

\section{References}

1. Ambard AJ, Mueninghoff LE. Calcium phosphate cement: review of mechanical and biological properties. Prosthodontics. 2006;15:321-8. https://doi.org/10.1111/j.1532-849X.2006. 001 29.x.

2. Chow LC. Next generation calcium phosphate-based biomaterials. Dent Mater. 2009;28:1-10. https://doi.org/10.4012/dmj.28.1.

3. Ritter Jones M, Messersmith PB. In situ forming biomaterials. Oral Maxillofac Surg Clin N Am 2002;14:29-38. https://doi.org/ 10.1016/s1042-3699(02)00015-8.

4. Zhang J, Liu W, Schnitzler V, Tancret F, Bouler JM. Calcium phosphate cements for bone substitution: chemistry, handling and mechanical properties. Acta Biomater. 2014;10:1035-49. https:// doi.org/10.1016/j.actbio.2013.11.001.

5. Habraken WJ, Liao HB, Zhang Z, Wolke JG, Grijpma DW, Mikos $\mathrm{AG}$, et al. In vivo degradation of calcium phosphate cement 
incorporated into biodegradable microspheres. Acta Biomater. 2010;6:2200-11. https://doi.org/10.1016/j.actbio.2009.12.028.

6. Ooms EM, Wolke JG, van der Waerden JP, Jansen JA. Trabecular bone response to injectable calcium phosphate (Ca-P) cement. J Biomed Mater Res. 2002;61:9-18. https://doi.org/10.1002/jbm. 10029.

7. Shie MY, Chen DC, Wang CY, Chiang TY, Ding SJ. Immersion behavior of gelatin-containing calcium phosphate cement. Acta Biomater. 2008;4:646-55. https://doi.org/10.1016/j.actbio.2007. 10.011 .

8. Carey LE, Xu HH, Simon CG, Takagi S, Chow LC. Premixed rapid-setting calcium phosphate composites for bone repair. Biomaterials. 2005;26:5002-14. https://doi.org/10.1016/j.biomateria 1s.2005.01.015.

9. Liu H, Li H, Cheng W, Yang Y, Zhu M, Zhou C. Novel injectable calcium phosphate/chitosan composites for bone substitute materials. Acta Biomater. 2006;2:557-65. https://doi.org/10.1016/j. actbio.2006.03.007.

10. Di Martino A, Sittinger M, Risbud M. Chitosan: a versatile biopolymer for orthopaedic tissue-engineering. Biomaterials. 2005;26:5983-90. https://doi.org/10.1016/j.biomaterials.2005.03. 016.

11. Barralet JE, Wang L, Lawson M, Triffitt JT, Cooper PR, Shelton RM. Comparison of bone marrow cell growth on $2 \mathrm{D}$ and $3 \mathrm{D}$ alginate hydrogels. J Mater Sci Mater Med. 2005;16:515-9. https://doi.org/10.1007/s10856-005-0526-z.

12. Xu HH, Takagi S, Quinn JB, Chow LC. Fast-setting calcium phosphate scaffolds with tailored macropore formation rates for bone regeneration. J Biomed Mater Res A. 2004;68:725-34. https://doi.org/10.1002/jbm.a.20093.

13. Unezaki Y, Eto T, Inoue H, Minamigawa K, Sugihara F. A study of hardening material consisted of tetracalcium phosphate dicalcium phosphate dehydrate and citric acid. J Jpn Prosthodont Soc. 1993;37:61-6.

14. Tonnesen $\mathrm{HH}$, Karlsen J. Alginate in drug delivery systems. Drug Dev Ind Pharm. 2002;28:621-30. https://doi.org/10.1081/ddc120003853.

15. Oh SA, Lee GS, Park JH, Kim HW. Osteoclastic cell behaviors affected by the a-tricalcium phosphate based bone cements. J Mater Sci Mater Med. 2010;21:3019-27. https://doi.org/10.1007/ s10856-010-4152-z.
16. Stankewich CJ, Swionthowski MF, Tencer AF, Yetknler DN, Poser RD. Augmentation of femoral neck fracture fixation with an injectable calcium phosphate bone mineral cement. J Orthop Res. 1996;14:786-93. https://doi.org/10.1002/jor.1100140516.

17. Ishikawa K. Calcium phosphate bone cement. In: Kokubo T (ed). Bioceramics and their clinical applications. Boca Raton, FL: Wood head Publishing in Materials; 2008. p. 438-63.

18. Xu HH, Wang P, Wang L, Bao C, Chen Q, Weir MD. et al. Calcium phosphate cements for bone engineering and their biological properties. Bone Res. 2017;5:17056. https://doi.org/10. 1038/boneres.2017.56.

19. Hu G, Xiao L, Fu H, Bi D, Ma H, Tong P. Study on injectable and degradable cement of calcium sulphate and calcium phosphate for bone repair. J Mater Sci Mater Med. 2010;21:627-34. https://doi. org/10.1007/s10856-009-3885-z.

20. Ishikawa K, Asaoka K. Estimation of ideal mechanical strength and critical porosity of calcium phosphate cement. J Biomed Mater Res. 1995;29:1537-43. https://doi.org/10.1002/jbm. 820291210 .

21. Ishikawa K, Takagi S, Chow LC, Ishikawa Y. Properties and mechanisms of fast-setting calcium phosphate cements. J Mater Sci Mater Med. 1995;6:528-33. https://doi.org/10.1007/ BF00151034.

22. Miyamoto Y, Ishikawa K, Fukao H, Sawada M, Nagayama M, Kon $\mathrm{M}$, et al. In vivo setting behavior of fast-setting calcium phosphate cement. Biomaterials. 1995;16:855-60. https://doi.org/ 10.1016/0142-9612(95)94147-d.

23. Tsuru K, Ruslin, Maruta M, Matsuya S, Ishikawa K. Effects of the method of apatite seed crystals addition on setting reaction of atricalcium phosphate based apatite cement. J Mater Sci Mater Med. 2015;26:244. https://doi.org/10.1007/s10856-015-5570-8.

24. Ginebra MP, Espanol M, Montufar E, Perez RA, Mestres G. New processing approaches in calcium phosphate cements and their applications in regenerative medicine. Acta Biomater. 2010;6:2863-73. https://doi.org/10.1016/j.actbio.2010.01.036.

25. Lee GS, Park JH, Won JE, Shin US, Kim HW. Alginate combined calcium phosphate cements: mechanical properties and in vitro rat bone marrow stromal cell responses. J Mater Sci Mater Med. 2011;22:1257-68. https://doi.org/10.1007/s10856-011-4296-5.

26. Teitelbaum SL. Bone resorption by osteoclasts. Science. 2000;289:1504-8. https://doi.org/10.1126/science.289.5484.1504. 